\title{
Endeavors in Micro-Imaging Spectrometry
}

\author{
M. M. Rosen
}

\section{RECEIVED \\ NoV 171995 \\ OSAI}

October 19, 1995

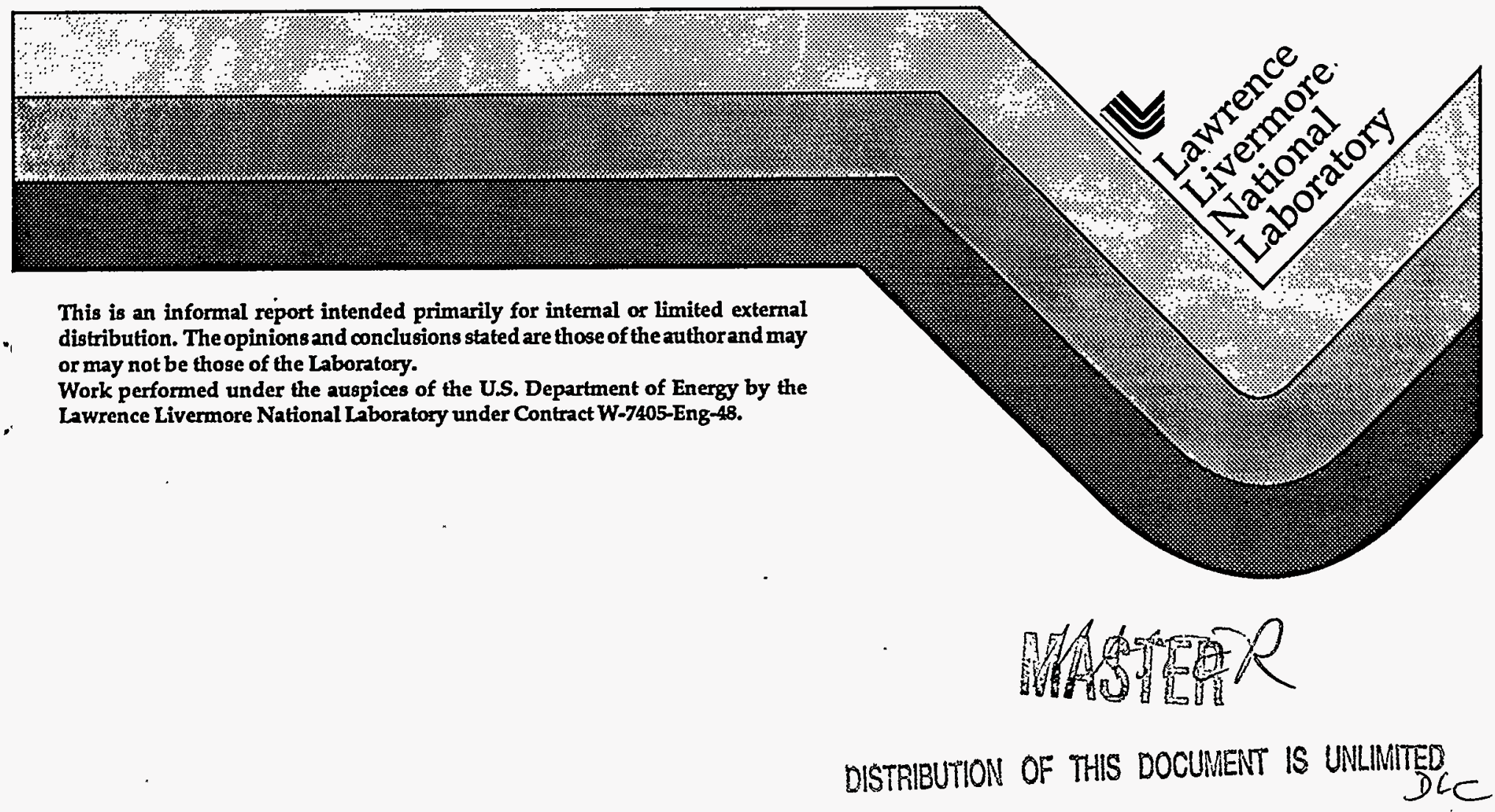


This document was prepared as an account of work sponsored by an agency of the United States Government. Neither the United States Government nor the University of California nor any of their employees, makes any warranty, express or implied, or assumes any legal liability or responsibility for the accuracy, completeness, or usefulness of any information, apparatus, product, or process disclosed, or represents that its use would not infringe privately owned rights. Reference herein to any specific commercial product, process, or service by trade name, trademark, manufacturer, or otherwise, does not necessarily constitute or imply its endorsement, recommendation, or favoring by the United States Government or the University of California. The views and opinions of authors expressed herein do not necessarily state or reflect those of the United States Government or the University of California, and shall not be used for advertising or product endorsement purposes.

This report has been reproduced directly from the best available copy.

Available to DOE and DOE contractors from the Office of Scientific and Technical Information P.O. Box 62, Oak Ridge, TN 37831

Prices available from (615) 576-8401, FTS 626-8401

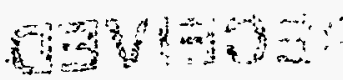

Available to the public from the National Technical Information Service

U.S. Department of Commerce

5285 Port Royal Rd.

Springfield, VA 22161

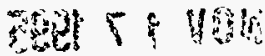

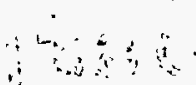




\section{Endeavors in Micro-imaging Spectrometry}

Michael M. Rosen

30 August 1995

The goal of this apparatus is to better enable characterization of tissue samples both on a microscopic scale and across the visible spectrum. The set-up consists of a phase-contrast inverted Nikon microscope, a single-grating imaging spectrometer, a CCD camera, and a computer potentially controlling all three of the previous devices. The computer uses an object-oriented program development environment called LabVIEW to run the three devices: This apparatus will hopefully enable better, less invasive surgical procedures, as well as permitting higher-resolution, more up-close observation of cellular dynamics.

\section{Apparatus}

The Nikon Diaphot microscope (Fig. 1A) has both transmitted and reflected light capabilities, as tissue samples exhibit different properties when observed through transmitted as opposed to reflected light. The microscope contains several apochromatic objectives which will provide up to 400 times amplification of the sample. An incandescent bulb is used to illuminate the sample and provide a white light source. The image is relayed out the side of the microscope and through a Nikon macro lens (Fig. 1B). This lens will refocus the image onto the slit of the spectrometer.

We have been using a Kaiser Optical Systems HoloSpec holographic imaging spectrometer (Fig. 1C). The spectrometer consists of a slit, 20 microns wide and about $5 \mathrm{~mm}$ tall, which allows in only a small area of the sample image. Once inside the spectrometer, the image is focused into a grating, or mirrorlike silver plate with thousands of grooves per millimeter. This grating will break up the light coming from the sample into its respective spectral elements. The particular spectrometer we are now using will be able to disassemble the light over the entire visible spectrum 
(wexhad earlier been using an older spectrometer which only allowed for a range of $70 \mathrm{~nm}$ at a time; the present one is calibrated to display light ranging from $392-790 \mathrm{~nm}$, or a range of almost 400 $\mathrm{nm})$. This fragmented light will be bounced off a mirror and refocused onto the CCD Camera (Fig. 1D).

A CCD (Charged Coupled Device) is simply a chip that is connected, via a GPIB (General Purpose Interactive Bus) cable to the computer. The chip consists of 1000 by 1024 pixels; each pixel consumes 2 bytes. Hence, use of the entire chip will cost the user two $\mathrm{Mb}$. The CCD Camera essentially uses this chip in place of film. So when the shutter of the camera is opened, the CCD is exposed to the refocused, broken-up light of the image coming from the spectrometer. This image is then relayed to the computer (Fig. 1E), and the LabVIEW program for analysis.

The program, known as "Camera Control V", requires as an input the number of pixels desired. The user can specify number of columns, rows, first column, and first row (Fig. 2C); to glean data from exactly the desired places on the chip. The user also controls the length of time that the camera shutter is left open (Fig. 2D), which will vary the amount of light allowed into the camera. After setting these options, the user can open the shutter, using the "start" button on the program control panel (Fig. 2E), and hence expose the chip. The data makes its way to the computer and within 30 seconds (for only one quarter of the whole chip, or 1024 by 256 pixels) a picture of the chip can be displayed on the control panel (Fig. 2A). This is a three-dimensional picture, two of the dimensions being the "coordinates" of the chip, the other being light intensity. The ydirection on the chip corresponds to wavelength, as the picture on the chip is simply the slit of the sample being expanded along the spectral range of the spectrometer. Hence, what we are most interested in is the relationship between this $y$-plane and the $z$ plane: intensity. We would thus like to hold the $x$-plane constant and make a two-dimensional graph. This process is similar to taking a cross-section of a cube (three dimensions) and displaying this square (two dimensions). This two-dimensional graph is displayed as the second graph on the control panel, known as a "lineout" (Fig. 2B). The program leaves the option open of taking several lines out from the three-d picture and displaying graphs of light intensity by wavelength. In general, troughs of intensity in these lineout graphs indicate peaks in light absorbance. We are thus able, roughly, to locate the proper wavelengths for the peak absorbances of the sample. 
Of course, that which is measured by the eye on Camera Control V's control panel can only be rough. Hence, there is an option in the program to relay the data from both the original picture and the lineout picture to another file, for manipulation on more powerful computers. This option increases both precision and convenience.

We've encountered two chief problems while working with the program and the apparatus in general. The first problem involves expediency. The way the program is presently organized, it takes us 30 seconds to take one picture of one quarter of the CCD chip. If we wanted to use the entire chip, it would take us 2 minutes to receive the image. Now that is two minutes for only the amount of sample that fits within the (approximately) 20 micron by $5 \mathrm{~mm}$ area of the slit. If we have a sample that is about one square $\mathrm{cm}$ in area, we would have to take 1000 separate pictures to get data for the entire sample. On top of the 2000 minutes that it would take to do so, we would have to realign the sample under the microscope after every exposure, which would take a lot longer. Thus, there is some software written to control, using LabVIEW and another GPIB cable, a motorized stage for the microscope. A type of "scan" command could be added to the present Camera Control V program (see enclosed description of Stage Scan v.i.) to facilitate quicker viewing of the entire sample. This addition could cut down on a lot of alignment time, yet the problem of speed still exists, as it would still take at least 2000 minutes to view the entire square $\mathrm{cm}$ sample. Some more options for increasing efficiency include simply not viewing every image taken and rather relaying the data directly to the file and a workstation (since the viewing itself eats up a lot of time), increasing computer memory, or simply replacing the computer with another, more powerful one. Yet another option is using a different program language to run the apparatus, however LabVIEW, while not known for its speed, is very convenient and user-friendly.

Another major problem we've encountered has been the interference of the white light spectrum with the spectrum of the sample. This occurs because the incandescent bulb used as the source light for the sample has a spectrum of its own, so the pictures we see are not purely the intensity of the sample at different wavelengths, but rather the combined intensities of the sample and the white light used to illuminate it (Fig. 3B). It thus becomes necessary to scale the image we receive according to the white light spectrum. In order to do this, we must first measure the white light spectrum, then set its peak intensity equal to 1 (Fig. 3C), and scale the rest of the spectrum accordingly. We can then divide the intensity at each point along the sample's spectrum by that at the 
respective point of the white light spectrum and thus receive the proper intensity graph for the sample (Fig. 3D).

\section{Applications}

The graphs shown on Fig. Page 3 all relate to pictures taken of a suture (Fig. 3A). A suture is simply a thread used by surgeons to stitch together open parts of the body during surgery. In order to do so, however, the surgeon must perform a fairly invasive, timeconsuming surgery. One idea to improve this surgery is welding the ends of the suture together using a laser instead of tying them together. In order to maximize laser efficiency (amount of energy and time of exposure to laser light), we must find the wavelength light that the suture would absorb best. Thus, we would use our apparatus to find the ideal wavelength at which to weld the ends of the suture.

Another application we've tested has been tissue welding. This is a process that employs lasers and a protein-based glue to repair organ ruptures. The glue is heated by the laser, as are the ends of the tissue, and when they cool, the rupture will close up. Again, to get high laser productivity, we need to know the proper wavelength laser with which to "cook" the glue (i.e. the wavelength that absorbs the most light). Our apparatus has been and can be used to find that wavelength.

Other applications include finding the specific light that will increase the contrast between nerve and muscle in carpal tunnel surgery and general tissue characterization. Ultimately, we would like an apparatus similar to ours to see actual clinical use, but at this point we are focusing on completing and refining the setup and then putting it into research-oriented use. 
i

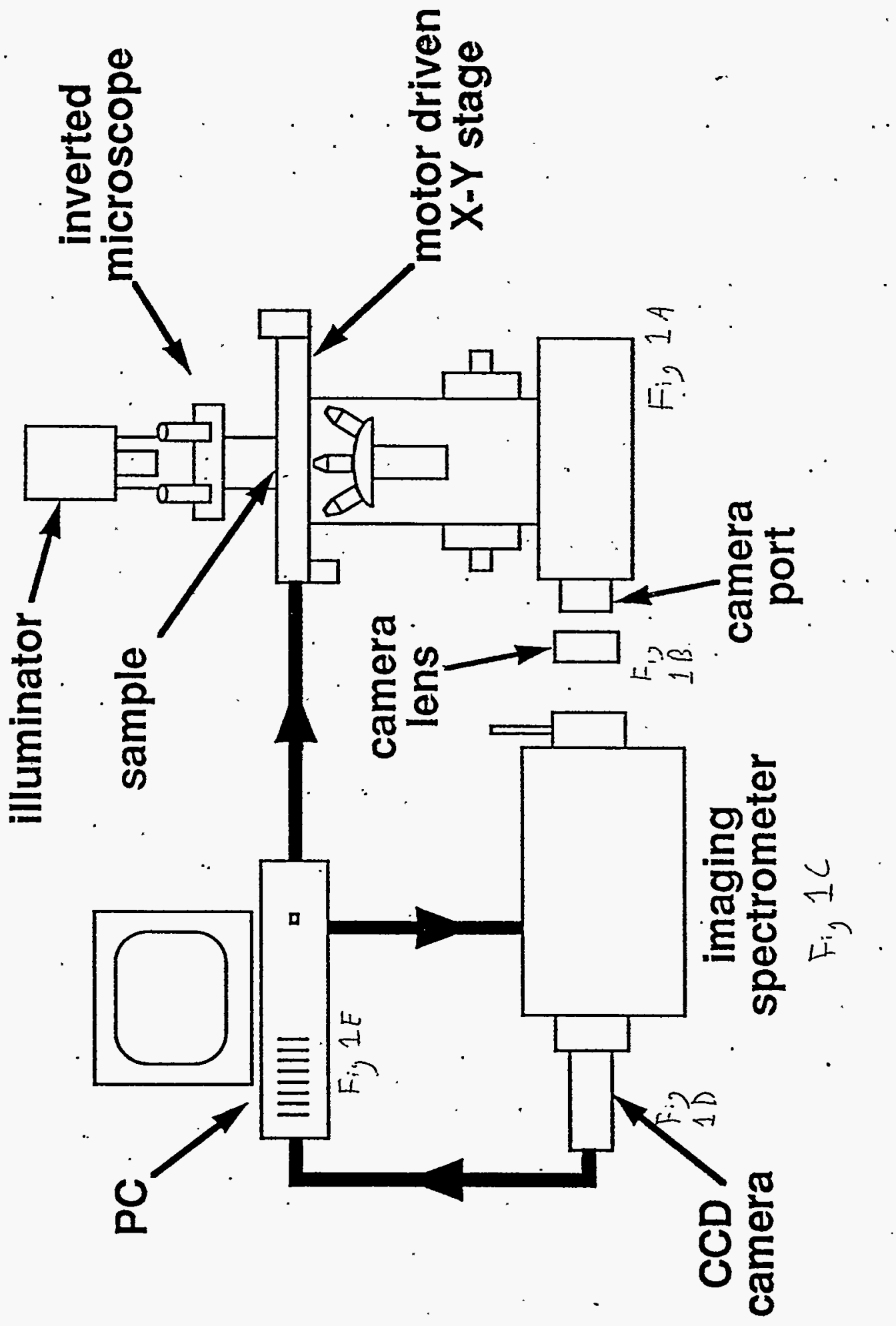



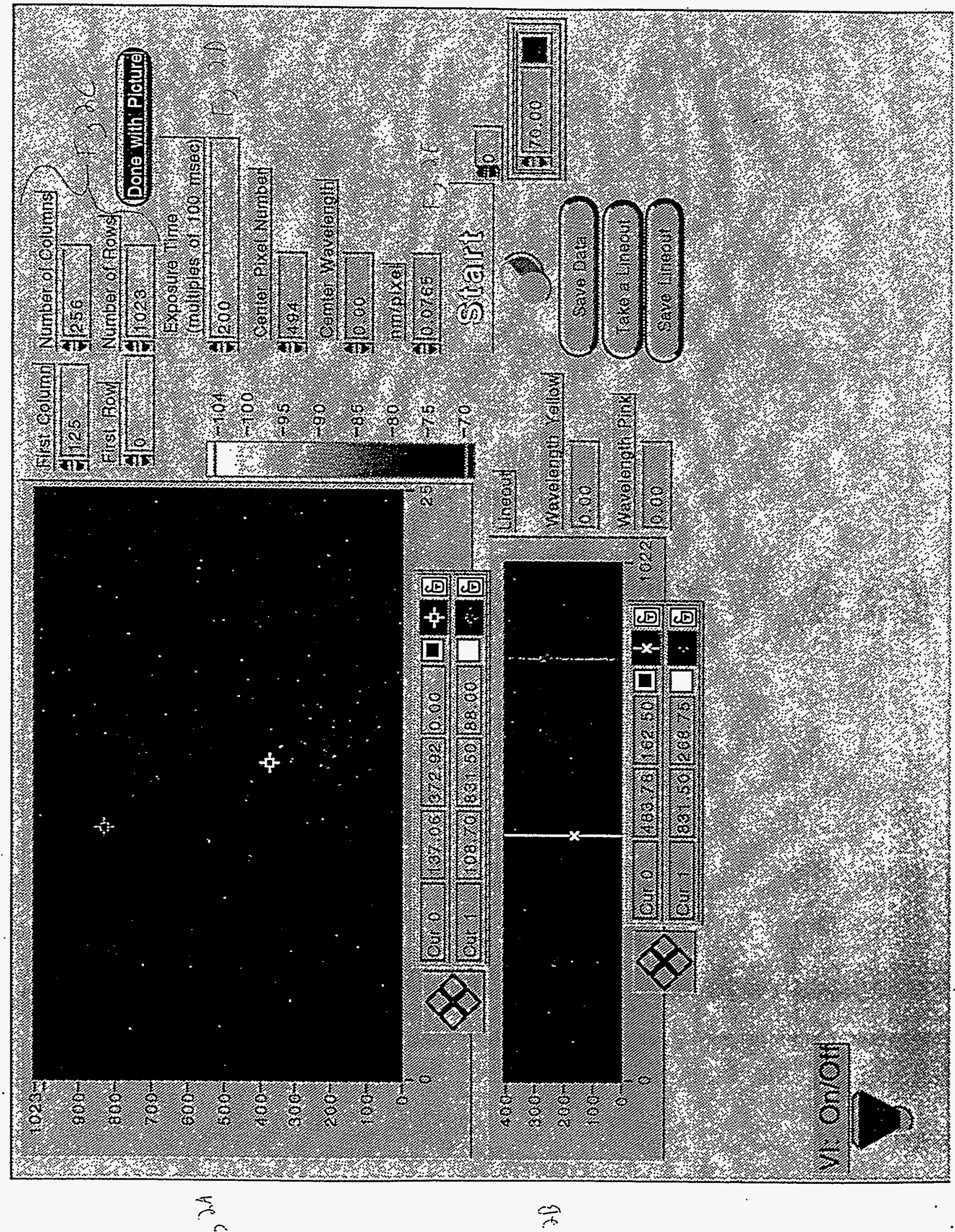


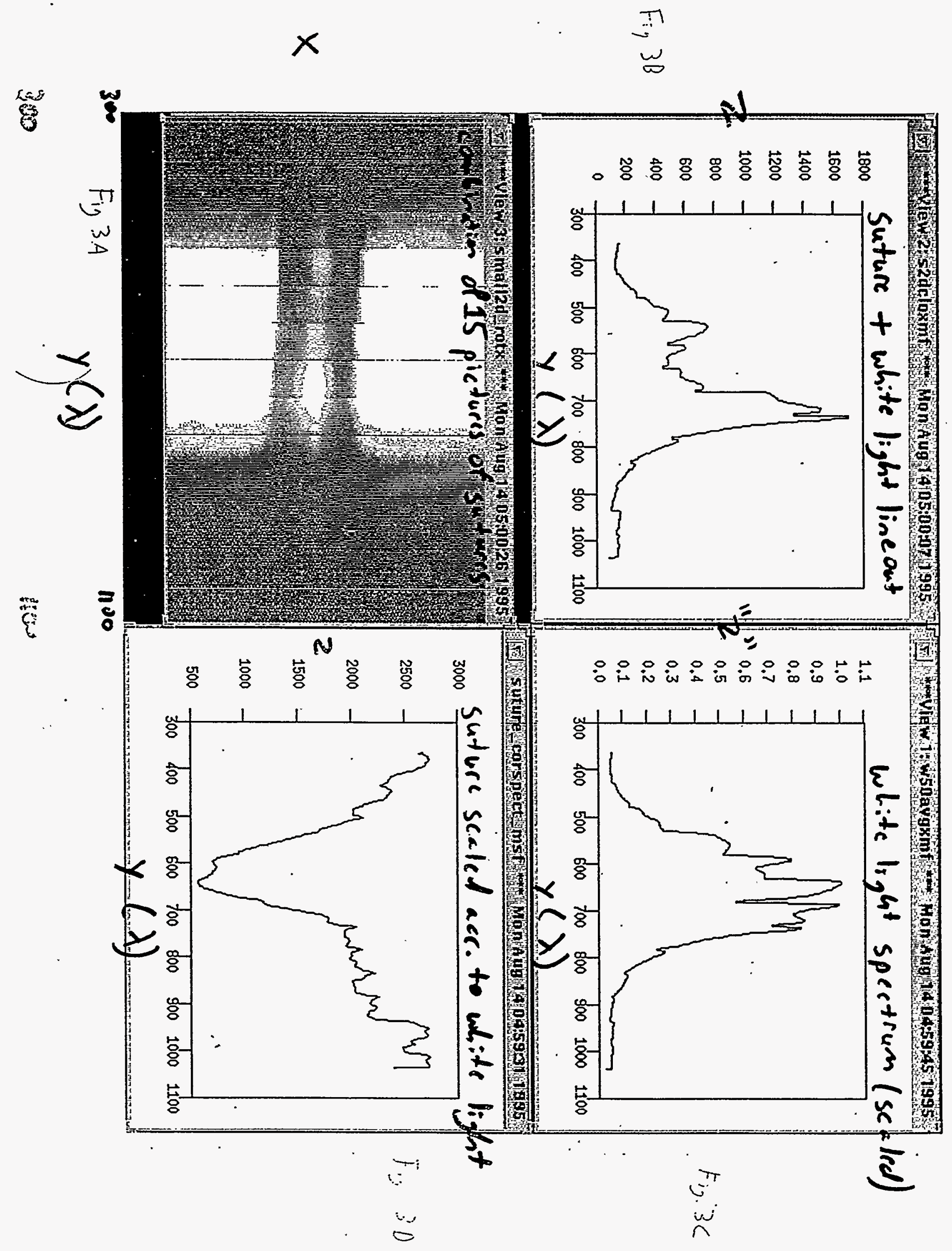




\section{Description of Camera Control V Front Panel}

31 August 1995

Michael M. Rosen

The front panel of the LabVIEW Camera Control V (CCV) "virtual instrument" (VI) enables the user to easily operate the CCD Camera that is part of the Micro-Imaging Optical Spectrometer (MIOS) apparatus. This sheet explains the functions on the front panel:

(Refer to LabVIEW manuals for more detailed information)

\section{1-5: CCV INPUTS}

1) First Column: the column number of the first column on the CCD chip to be displayed. There are 1000 numbered columns, from 0 to 999.

2) Number of Columns: the number of columns to be displayed.

3) First Row: the row number of the first row on the CCD chip to be displayed. There are 1024 numbered rows, from 0 to 1023.

4) Number of Rows: the number of rows to be displayed.

5) Exposure Time: the length of time ( $\mathrm{ms})$ that the shutter is open.

\section{6-10: CCV COMMANDS}

6) Start: trips shutter of CCD camera and opens it for amount of time specified.

7) Save Data: saves data relayed by CCD camera into file selected by user.

8) Take a Lineout: holds constant the x-value on Main Display Screen (display 11) of specified cursor (cosmetic device D) and graphs, on display 12, the z-scale (light intensity) by y-scale ( $y$-coordinate of CCD chip/ wavelength). User must employ cursor (cosmetic device D), or simply use mouse to move cursor.

9) Save Lineout: saves data contained in the lineout graph into file selected by user.

10) Done with Picture: terminates programmatic loop and disables saving and lineout capabilities. User should press button only when sure there is no more to be done with that particular exposure. 
11) Main Display Screen: displays three-dimensional image relayed over GPIB cable by CCD camera. Image on main display screen is that of CCD chip. $y$-axis is $y$-coordinate of CCD chip, $x$-axis is $x$-coordinate of CCD chip, and $z$-axis is light intensity, as seen on CCD chip. Âil axes autoscale according to data. On the y-axis, the up direction (toward 1000) corresponds to a DECREASE in wavelength (in the blue direction of the visible spectrum). Conversely, the down direction is an INCREASE in wavelength (toward the red). Wavelength information is displayed on Cosmetic Displays F and G. Image/display data can be saved using Save Data command (command 7).

12) Lineout Display Screen: displays two-dimensional image of "line" taken from Main Display Screen (display 11) according to cursor position (cosmetic device D). Cursor determines constant $x$-value (of Main Display) but $x$-axis of Lineout Display corresponds to $y$-axis of Main Display ( $y$-coordinate of CCD chip/ wavelength) and $y$-axis of Lineout corresponds to z-axis of Main (light intensity). Progress in out direction (toward 1022) along $x$-axis corresponds to a DECREASE in wavelength (toward the blue) and progress toward 0 is an INCREASE in wavelength (toward the red). This data can be saved using Save Lineout command (command 9).

\section{A-C: COSMETIC INPUTS (OBSOLETE?)}

These devices were used with the old spectrometer, and depended on the specified center wavelength of the spectrometer. With the new spectrometer, these inputs will most likely be unnecessary, as there will only be one center wavelength on the new spectrometer. These are changes in progress.

\section{D-E: COSMETIC DEVICES: CURSORS}

D) Main Display Screen cursors: These two cursors move in Main Display Screen and can be used both to pinpoint specific $x-, y-$, and $z$-values and to set a constant $x$-value for a lineout.

E) Lineout Display Screen cursors: These apply to Lineout Display (can be moved up and down to trace lineout graph), but are generally mere adjucts to cursors $D$. When cursor $D$ is moved up and down (y-plane) in Main Display, cursor $E$ moves side to side ( $x$-plane), as $y$-plane in Main Display corresponds to $\mathrm{x}$-plane in Lineout Display.

\section{F-G: COSMETIC DISPLAYS}

Using equations in program code, $y$-coordinate in Main Display data is converted to corresponding wavelength (using old spectrometer, this was dependent on specified center wavelength, but with new spectrometer; there will be fewer variables in these equations). Displays $F$ and $G$ will show wavelength corresponding to $y$-coordinate on which activated cursor (pink or yellow) is situated. 

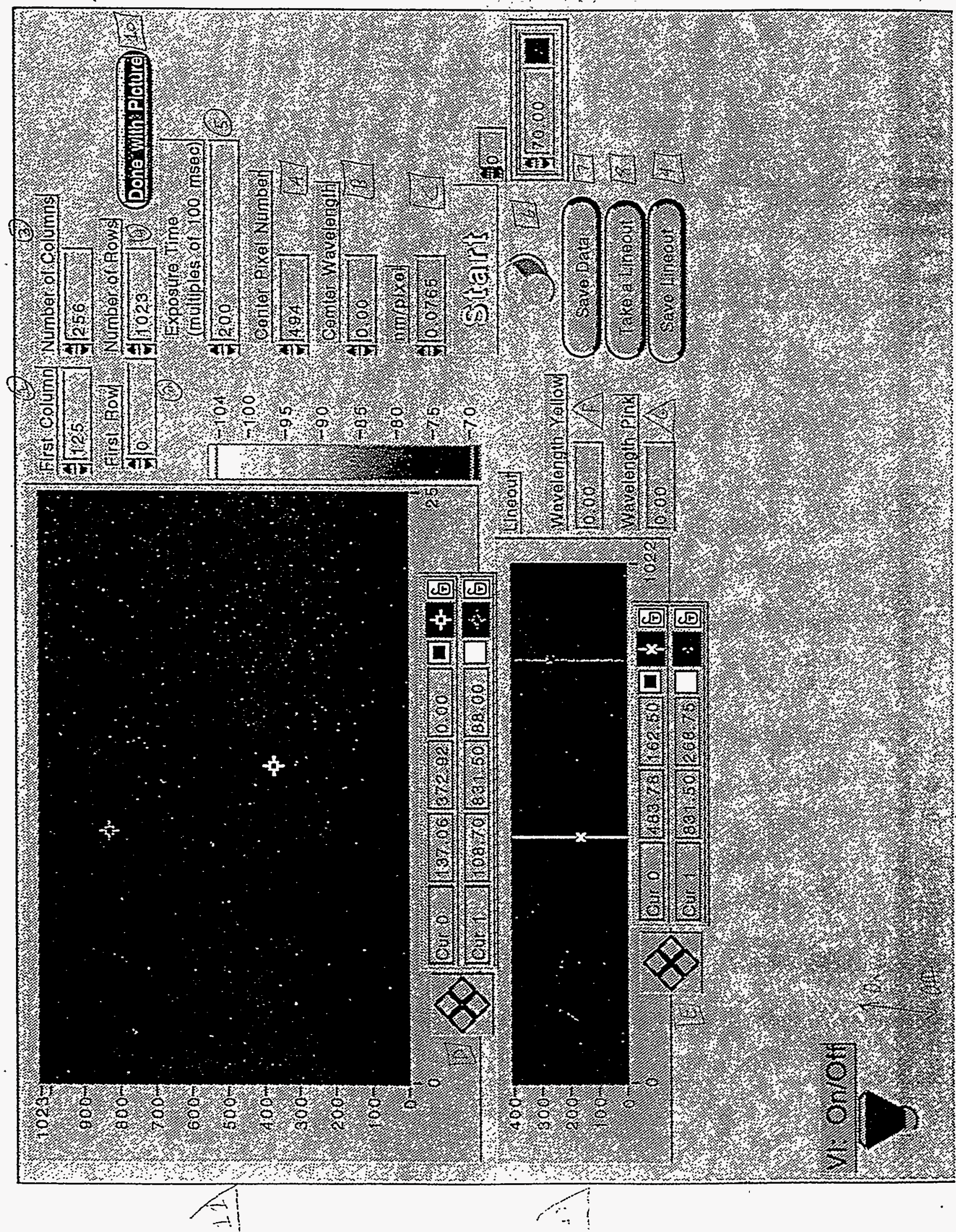


\section{Description of Stage Scan Front Panel}

31 August 1995

Michael M. Rosen

In order to expedite operation of the Micro-Imaging Optical Spectrometry apparatus, there has been software written (a LabVIEW virtual instrument called Stage Scan (SS)) to instruct motorized microscope stages to perform a scan of the tissue sample. This is a reference sheet to facilitate the use of that "v.i."

N.B. Due to the non-arrival of the stages during my term at the Lab, this v.i. has never been tested. There may be problems in its operation. The user is requested to consult with the manual enclosed with the motorized stages in order to modify the program so that it works.

\section{1) AUTOSCAN MODE INPUTS}

1A) Initial X-value: instructs stage where to begin scanning in $x$-plane.

1B) Initial Y-value: instructs stage where to begin scanning in y-plane.

1C) Number of Steps: number of increments in scanning process.

1D) Step Width: width of increment.

\section{2) MANUAL SCAN MODE INPUTS}

2A) Xstep: commands movement for absolute distance in $x$-plane.

2B) Ystep: commands movement for absolute distance in y-plane..

\section{3) ABSOLUTE POSITION INPUTS}

3A) Toxvalue: commands movement to absolute $x$-coordinate.

3B) Toyvalue: commands movement to absolute $y$-coordinate.

\section{4) SCAN SPEED INPUTS}

4A) X-speed: sets speed of motor moving in $x$-direction (motor $x$ ). 
4B) Y-speed: sets speed of motor moving in $y$-direction (motor y).

\section{5) SERIAL PORT INPUTS}

5A) Port Number: informs RS232 connection which port to connect to.

5B) Action: instructs RS232 board to either write inputted information from program or read outputted information from the stage.

\section{6-7) STRING-RELATED OUTPUTS}

6) Length of String: a non-crucial output; simply informs user how long string inputted to RS232 board was.

7) Concatenated String: describes to user what string looks like. 


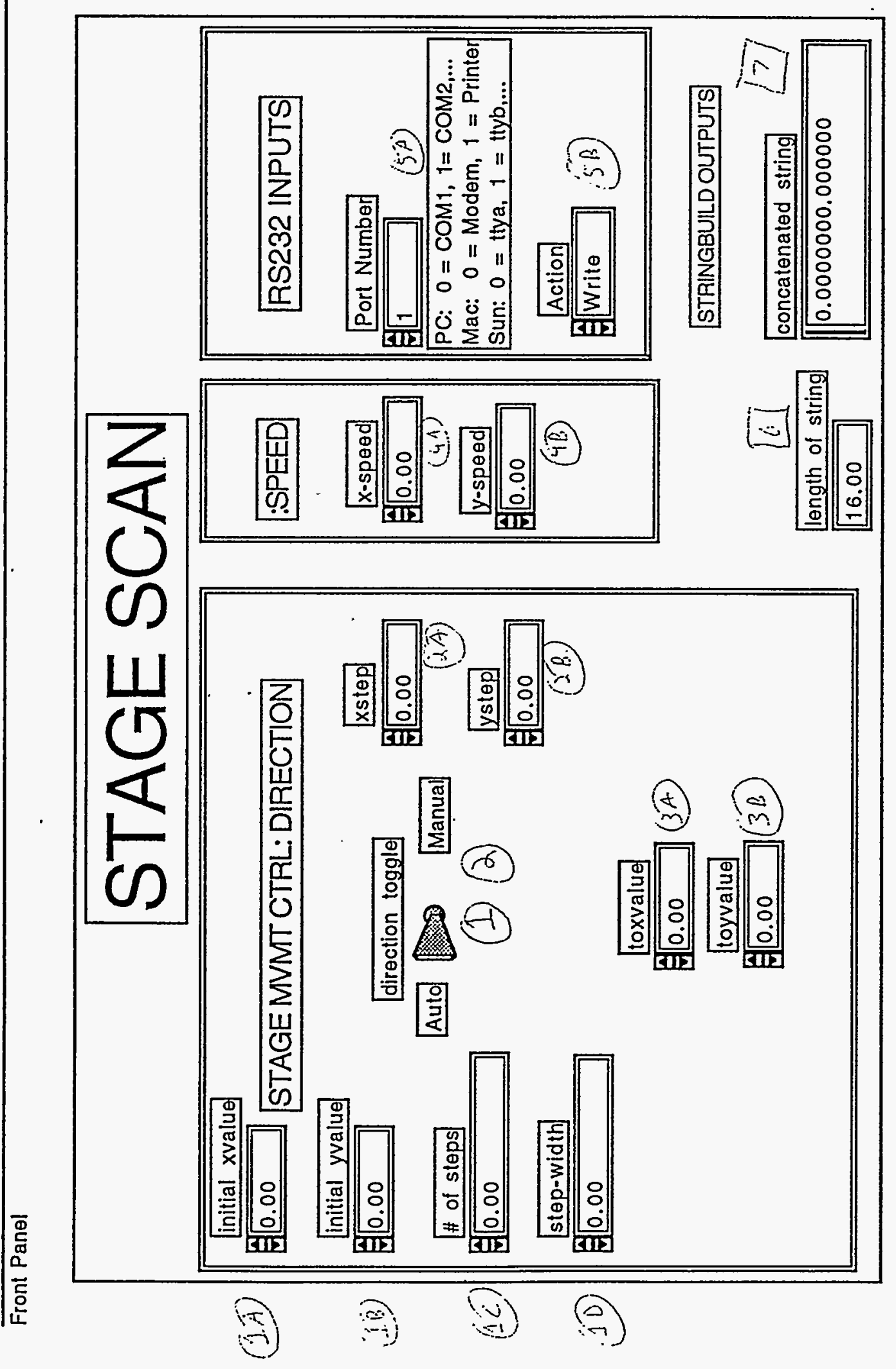




Technical Information Department - Lawrence Livermore National Laboratory

University of California • Livermore, California 94551

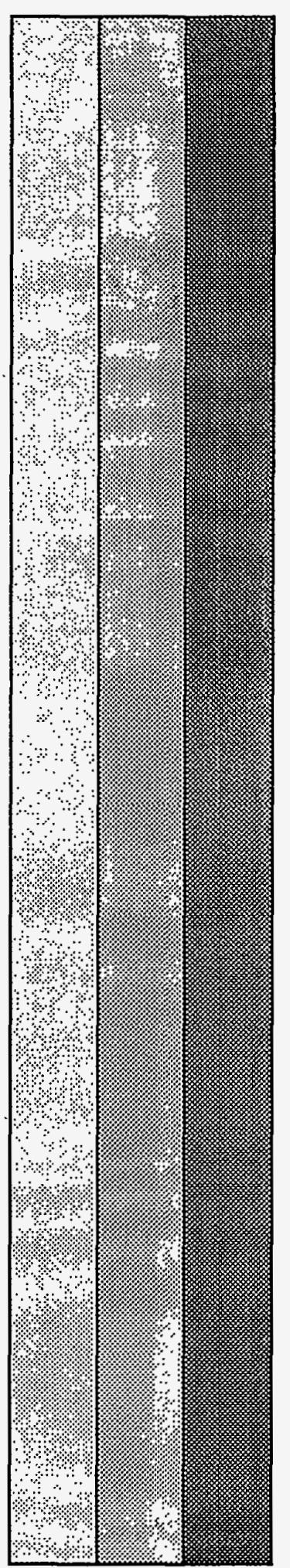

